\title{
DOS RECORRIDOS DIFERENTES \\ PARA LA IMPLANTACIÓN \\ DE LAS COMPETENCIAS \\ EN LA UNIVERSIDAD: ESPAÑA Y EE. UU.
}

\section{Two different routes for the implementation of the competences in the University: Spain and USA}

\author{
Iluminada SÁnchez Domenech \\ Universidad Internacional de La Rioja (Logroño) \\ Correo-e: iluminada.sanchez@unir.net \\ Mariano Rubia Avi \\ Universidad de Valladolid \\ Correo-e: mrubia@pdg.uva.es \\ Recepción: 22 de noviembre de 2015 \\ Envío a informantes: 24 de noviembre de 2015 \\ Aceptación definitiva: 6 de abril de 2016
}

RESUMEN: Este documento muestra los distintos recorridos que han seguido EE. UU. y España en la implantación del modelo de competencias, con lo que se pretende contribuir a una mejor comprensión del concepto "competencia» y a una visión crítica del enfoque al que se acusa de una visión reduccionista de la educación y de la persona. Analizamos los antecedentes del concepto y su desarrollo en EE. UU. Un análisis comparado de otros conceptos relevantes a través de los documentos Tuning revela ambigüedades y errores conceptuales en el europeo. Obviar la base conductual y la orientación a la rendición de cuentas del enfoque competencial ha llevado a omitir un paso esencial del diseño curricular: partir de unos objetivos de aprendizaje medibles. Cuestionamos lo «innovador» de las metodologías activas de aprendizaje, así como la estructura de la Universidad española y su cultura institucional como entorno propicio para los aprendizajes transversales.

Palabras clave: competencias; objetivos de aprendizaje; diseño curricular; educación superior; metodologías de enseñanza-aprendizaje; evaluación de competencias en educación superior.

Aвstract: This document shows the different routes that have followed us and Spain in the implementation of competency model. We want to contribute to a better 
understanding of the concept of «competency» and to adopt a critical view of the approach which is accused of a reductionist view of education and of the person. We analyze the history of the concept and its development in the us. A comparative analysis of other relevant concepts through the Tuning documents reveals ambiguities and misunderstandings in the European. To obviate the behavioral basis and orientation to the accountability of the competence approach has led to omit an essential step in curriculum design: to start from measurable learning objectives. We questioning what «innovative» are the active learning methodologies and the structure of the Spanish University and its organizational culture as supportive environment for cross learnings.

KEY WORDS: competencies; learning objetives; curricular design; higher education; teaching and learning methodologies; competencies assessment in higher education.

\section{Introducción}

$\mathrm{D}$ E ACUERdo CON DíAz BARriga (2009) dos aspectos que sobresalen cuando se lee la literatura en español sobre competencias son la confusión y la euforia innovadora. A pesar de que el informe Tuning (2006) intentaba proporcionar "potentes herramientas para mejorar la calidad del diseño e impartición de programas» (p. 8I), lo cierto es que no deja del todo claro cómo llevar a cabo el diseño curricular por competencias a juzgar por la abundancia de artículos e informes dedicados al tema.

El modelo de competencias en las universidades de EE. UU., aunque no ha sido impuesto abiertamente por el Gobierno central ni los gobiernos federales, ha suscitado algunas críticas que denuncian la presión para su implantación y el peligro que supone para la educación liberal ${ }^{\mathrm{I}}$ y para la democracia que caracteriza a su educación universitaria. En esta corriente crítica se sitúa Marta Nussbaum (2010), filósofa estadounidense ligada al modelo de Desarrollo Humano. La autora advierte que el modelo de educación orientado al crecimiento económico y la competitividad global tiende a descuidar aquellos aprendizajes que sostienen la vida democrática en favor de los aprendizajes técnicos o aplicados para la vida laboral:

Sedientos de dinero, los estados nacionales y sus sistemas de educación están descartando sin advertirlo ciertas aptitudes que son necesarias para mantener viva a la democracia. Si esta tendencia se prolonga, las naciones de todo el mundo en breve producirán generaciones enteras de máquinas utilitarias, en lugar de ciudadanos cabales con la capacidad de pensar por sí mismos, poseer una mirada crítica sobre las tradiciones y comprender la importancia de los logros y los sufrimientos ajenos. El futuro de la democracia pende de un hilo (p. 20).

En España, algunas voces críticas denuncian que la reducción de la educación superior a la formación en competencias para el mercado laboral global excluye la

Utilizamos la definición de la Association of American Colleges \& Universities (AAC\&U, 2016) del concepto americano de Educación Liberal: «Es un enfoque para el aprendizaje que empodera a los individuos y los prepara para hacer frente a la complejidad, la diversidad y el cambio. Provee a los estudiantes de un amplio conocimiento del mundo en general (ej. ciencia, cultura y sociedad), así como, un estudio profundo de un área específica de interés. [...] ayuda a los estudiantes a desarrollar tanto el sentido de la responsabilidad social, fuertes y transferibles habilidades intelectuales y prácticas, como las habilidades de comunicación, analíticas y de resolución de problemas y una demostrada capacidad para aplicar el conocimiento y las habilidades en ambientes del mundo real». 
formación humana y el espíritu crítico orientado a la mejora social, así como la instrumentalización de las competencias «interpersonales» a su utilidad profesional y económica (Solá, 2009).

El análisis de los documentos Tuning, europeo (2006) y americano (20I2), pone de manifiesto algunos errores conceptuales en el primero que han contribuido a la confusión, como equiparar resultados de aprendizaje y competencias, atribuyendo a estas últimas una objetividad falsa (Hager, 2006), o intentar desligar la competencia de su base conductual y su orientación a la rendición de cuentas (Hyland, 2006) cuando, en realidad, son indisociables. Los últimos documentos publicados por Tuning (Wagenaar, 20I4) y ANECA (Agencia Nacional de Evaluación de la Calidad y Acreditación, 20I4/20I5) reconocen estas ambigüedades u omisiones de los anteriores.

Este documento muestra los distintos recorridos que han seguido EE. UU. y España en la implantación del modelo de competencias tal como se está llevando a cabo en la Universidad española y en la estadounidense. Nuestros objetivos son: en primer lugar, una mejor comprensión del concepto de competencia a través de su origen dentro de la psicología laboral estadounidense y, en segundo lugar, una visión crítica del enfoque que se revela por sí misma, sin que sea necesaria más explicación, al constatar el verdadero origen y objetivo del modelo y al compararlo con el estadounidense.

\section{Antecedentes conceptuales del modelo de competencia en EE. UU.}

Como declara uno de los artífices del informe Tuning europeo, Wagenaar (20I4), «Tuning es bien consciente de que el lenguaje elegido se originó fuera de lo académico» (p. 26). Veamos cuáles fueron esos orígenes extraacadémicos.

\section{I. De «incidente critico» a "competencia»}

La psicología conductista venía colaborando desde la II Guerra Mundial con el departamento militar de EE. UU. para formar de forma eficiente a los soldados en el manejo del armamento (Hodge, 2007), con el Departamento de Estado para la selección y formación de diplomáticos (es el caso de McClelland según Goleman, 1998), con las Fuerzas Aéreas en la formación de pilotos (Flannagan, 1954), etc. Estos dos autores, Flannagan y McClelland, realizan con sus investigaciones importantes aportaciones al concepto e identificación de las competencias.

En 1954 Flanagan presenta en un artículo para el Psychological Bulletin titulado la «La técnica de incidentes críticos» que «consiste en una serie de procedimientos para recoger observaciones directas de comportamiento humano de tal forma que facilite su uso potencial para resolver problemas prácticos y desarrollar principios psicológicos generales» (Flanagan, 1954:327). Flanagan entiende por «incidente» cualquier actividad humana que es suficientemente completa en sí misma como para permitir hacer inferencias y predicciones sobre el desempeño de la persona que realiza la acción. Para ser crítico, un incidente debe ocurrir en una situación donde el propósito o la intención de la acción aparezca suficientemente clara para el observador y donde las consecuencias sean suficientemente definidas de forma que dejen pocas dudas sobre sus efectos. 
Los informantes (alumnos, clientes, pacientes, supervisor) describían situaciones en las que la persona observada (profesor, médico, empleado) se había comportado de forma que destacaba por un desempeño fuera de la norma tanto en sentido positivo como negativo. Flanagan no llegó a hablar de «competencias», sino de «comportamientos clave» para un buen o mal desempeño en un puesto trabajo determinado. Tampoco llegó a proponer métodos para formar o aprender esos comportamientos, sino para identificarlos en los empleados. Su técnica sirvió de base a McClelland para desarrollar un método para identificar competencias asociadas a un puesto de trabajo.

De acuerdo con Wagenaar (20I4) el concepto fue por primera vez relacionado con la educación por McClelland en 1973. En ese año McClelland publica en la revista American Psychologist el artículo «Testing for Competence Rather Than for "Intelligence”». En el artículo McClelland arremete contra los test de inteligencia estandarizados utilizados para etiquetar a alumnos en las escuelas, así como por las universidades para seleccionar a los alumnos con más altas puntuaciones. Propone como alternativa sustituir «inteligencia» por conceptos como «potencial», frente a lo que una persona es capaz de hacer en el presente, y "competencia», basada en comportamientos relevantes y observables. Como alternativa a los test estandarizados propone en este artículo seis criterios o principios para construir métodos de evaluación que pongan a prueba las «competencias», de los que destacamos sólo tres por razones de espacio:

- La mejor prueba es el criterio de muestreo: «Si quieres probar quién será un buen policía, ve a averiguar qué hace un policía, síguelo a todas partes, haz una lista de sus actividades y muestrea esa lista en la selección de candidatos» (p. 7), teniendo en cuenta que parte del muestreo de trabajo tendrá que basarse tanto en conocimientos teóricos como en la práctica.

- La prueba debe evaluar las competencias agrupadas por situaciones de la vida. En este nuevo enfoque que propone McClelland, hay dos tipos de competencias: las específicas de una profesión determinada y otras más generales y deseables en situaciones sociales como liderazgo, habilidades interpersonales, habilidades de comunicación, paciencia, establecer metas moderadas, desarrollo del ego o desarrollo moral, etc.

- La definición de objetivos conductuales en la aplicación de pruebas en el ámbito laboral puede producir enormes listas de inventarios de comportamientos de pequeñas habilidades con escaso poder predictivo general, por lo que recomienda formularlos en términos de códigos de pensamiento con una abstracción conductual más amplia con aplicabilidad a un número mayor de situaciones.

Para concluir su artículo, McClelland (1973) hace algunas recomendaciones para la educación. Una de ellas se refiere a otro tipo de características, el desarrollo del ego y el desarrollo moral, que, según el autor, debe desarrollar también el sistema de educación superior aunque esta vez se refiere a ellas como «características» y no como «competencias».

Fruto de su colaboración con el Departamento de Estado para la selección y formación de diplomáticos, McClelland concluyó, según Goleman (I998), que: (I) las duras pruebas académicas a las que se sometía a los candidatos para ocupar un puesto en el Departamento de Exteriores se correlacionaban negativamente con su desempeño en las pruebas de selección. El desempeño se relacionaba con las competencias que 
se necesitaban en la acción; (2) lo que realmente distinguía a los más brillantes de sus compañeros mediocres giraba en torno a una serie de habilidades humanas básicas.

\subsection{El desarrollo del concepto de competencia en el trabajo}

En EE. UU. la CBE se lleva utilizando desde los años 70 en la formación profesional. Así, en 1979 Morrison recomendaba en una guía elaborada para el Departamento de Educación de Pensilvania sobre la formación ocupacional de adultos este nuevo enfoque:

Un movimiento importante de rápido crecimiento en la educación de hoy es la educación basada en competencias ( $\mathrm{CBE}$ ). Un programa que se define por competencias es el que proporciona un enfoque sistemático a la instrucción que satisfaga las demandas de rendición de cuentas. Implica el diagnóstico de lo que sabe cada alumno ${ }^{2}$, identificando lo que cada alumno necesita saber para cumplir con las normas de ingreso en el trabajo de los empresarios de la comunidad, que prescribe la forma en que el alumno va a lograr estos objetivos, y luego evalúa el resultado para asegurarse de que todos los objetivos se han cumplido. En la preparación para el trabajo, el uso de este enfoque proporciona un estándar para medir el nivel de competencia de cada estudiante al terminar un programa (Morrison, 1979: 2).

Por tanto, este nuevo enfoque aportaba dos novedades: I) objetivos de aprendizaje evaluables reformulados en términos de competencias demandadas por los empleadores y 2) rendición de cuentas.

Dos alumnos de McClelland, Boyatzis y Goleman, seguirán la investigación de las competencias en ambientes laborales contribuyendo al desarrollo de su conceptualización y de la metodología para su identificación. Boyatzis (1982) estudia las competencias de 2000 personas y 4I gerentes que trabajan en 12 organizaciones diferentes, públicas y privadas. Su posición en una gran consultora, McBer and Company, le permite el estudio experimental que le lleva tanto a un desarrollo del concepto de competencia profesional como a un método para su identificación y evaluación. Éste consta de una serie de pasos en los que no nos detendremos por razones de espacio. El tercer paso consiste en una «forma de entrevista de incidentes críticos (Flanagan, 1954) que McClelland llama Behavioral Event Interviewing»(Boyatzis, 2008: 4I). El BEI consiste en una entrevista en profundidad que es grabada para su posterior análisis. El entrevistado es preguntado sobre lo que hace, piensa y siente en una determinada situación laboral (Goleman, I998).

Según Boyatzis (2008), las competencias son una «aproximación conductual a la inteligencia emocional, social y cognitiva» (p. 7). Pero "para definir una competencia es necesario determinar cuáles fueron las acciones y su ubicación en un sistema y secuencia de comportamento y cuáles fueron sus resultados y efectos y cuál fue la intención y significado de las acciones y sus resultados» (p. 22). Un elemento que a menudo se pasa por alto cuando se trata de definir las competencias es la «intención»: en una situación en la que una persona escucha a otra y le pregunta por sus asuntos, la intención de esa persona puede ser captar la simpatía de la otra persona o aparentar interesada

El diagnóstico de las competencias que ya posee el alumno fruto de la experiencia profesional o personal (Prior Learning Assessment o PLA) es una práctica recomendada en EE. UU. en la educación de adultos vocacional. 
ganando así prestigio o, por el contrario, puede escuchar y preguntar porque realmente le interesa comprender a la otra persona, conocer sus prioridades y lo que piensa de una situación. En este último caso se trata de una demostración de empatía. En el primero se trata de una demostración de influencia. Es decir, la competencia observable sería la empatía, sin embargo, lo que lleva a la persona al comportamiento empático posee un sustrato moral, que no se puede observar externamente y que determina la intención.

Boyatzis (1982) incluye el concepto de "puesto de trabajo» poniendo de relieve así que la misma competencia, aunque tenga el mismo nombre, no significará lo mismo en distintas profesiones. También, la «organización concreta» determina a través de sus valores, cultura, misión y estrategia las competencias que considera adecuadas para cada puesto de trabajo. Se trata, pues, de un modelo sistémico que se representa en la Figura I.

Figura i: Teoría de la acción y el rendimiento en el trabajo

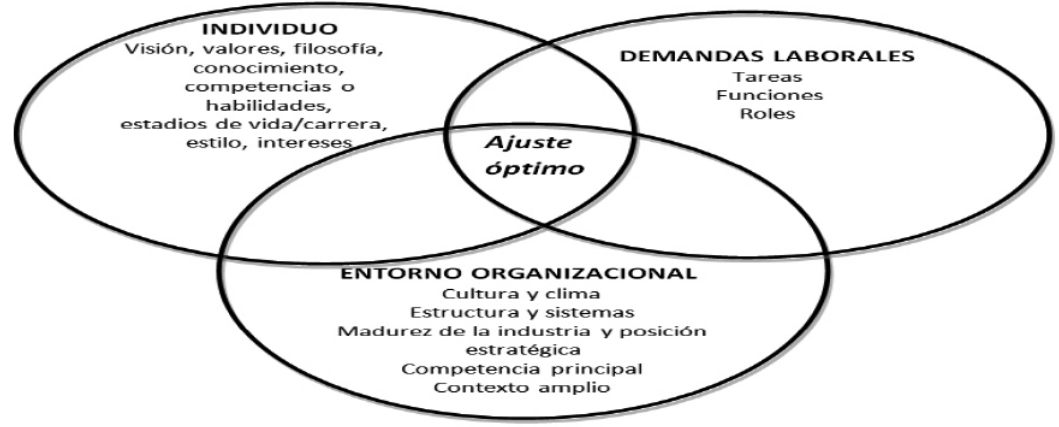

Fuente: Boyatzis (2008: 8).

Destaquemos algunas conclusiones relevantes para el concepto de competencia: (I) la competencia, entendida como comportamiento excelente de un empleado en un puesto de trabajo y organización concretos, se manifiesta e identifica sólo a través de la observación en determinadas situaciones críticas de la vida laboral real; (2) a pesar de que la formación o la educación iniciales se dirijan a formar las competencias supuestamente demandadas por el mercado laboral, habrá otras que el individuo deberá desplegar o inhibir, mediante la formación y la experiencia en la empresa, para que el ajuste entre el empleado - sus valores, competencias, estadio de desarrollo de la carrera, etc. - y la misión, valores, estrategia, clima y cultura, clientes, etc., de la organización sea el óptimo para que se manifiesten las competencias deseables en ese puesto concreto; (3) una cosa es identificar o evaluar competencias que ya posee el individuo, en una situación laboral real, y otra muy distinta conocer cómo las ha adquirido y ayudar a otras personas a adquirirlas o formarlas. El proceso de aprendizaje mediante el cual se adquirió la competencia no se contempla ni en el origen ni en el desarrollo concepto; (4) por otra parte, Boyatzis, Goleman y Rhee (2000) apuntan a que existe una fuerte relación entre la agrupación de las competencias (aquellas que se manifiestan juntas) y la filosofía de funcionamiento o los fundamentos de valor 
(que no son tratados como competencias) que sostiene una persona, así como sus rasgos de personalidad y su motivación y que, entre otras cosas, determinará la «intención» de la competencia.

\section{La competencia en la educación: EE. UU. y España}

El salto de las competencias a la educación universitaria en EE. UU. no ha sido automático aunque las pruebas estandarizadas sí fueron adoptadas en los niveles inferiores. Actualmente, todavía la mayoría de universidades estadounidenses no han adoptado el modelo de competencias, algunas se encuentran en un estadio híbrido (CAEL [Council for Adult and Experiential Learning], 20I2), que abordamos más adelante, entre la formación basada en cursos y las competencias.

En EE. UU. Tuning ha sido financiado por las entidades privadas Lumina Foundation y la William and Flora Hewlett Foundation. En el documento Tuning American Higher Education: The Process elaborado por el IEBC (Institute Evidence-Based Change) en el 2012 para estas entidades, se destacan las diferencias que caracterizan el proceso Tuning en EE. UU. con respecto al proceso Tuning en Europa y que determinan el desarrollo e implementación del proceso: i) Debe ser implantado antes del bachillerato para adaptarse a la estructura del sistema educativo, extendiéndose también a los niveles inferiores que dan acceso a la universidad para hacer efectivo un aprendizaje progresivo de una disciplina determinada. 2) Tuning pretende mantener las características de la educación superior estadounidense: amplia educación liberal, aprendizaje especializado con aplicación social relevante, educación cívica para una democracia caracterizada por una sociedad plural y metodologías instructivas integradas, holísticas y focalizadas en la investigación y en el aprendizaje centrado en el estudiante. 3) Debido a que no existe un gobierno federal centralizado de educación como en Europa (esto lo dice el Tuning americano, no nosotros), los sistemas estatales son los que han desarrollado los proyectos pilotos de Tuning en EE. UU. (Indiana, Minnesota, Utah, Texas y Kentucky).

En España parece un lugar común en el ámbito universitario situar el origen de las competencias en la educación en el informe de Delors y colaboradores para la UNESCO, conocido en España con el título La educación encierra un tesoro (UNESCO, 1996). En el informe, como es sabido, los autores proponen cuatro pilares del conocimiento en los que debe estructurarse la educación: aprender a conocer, aprender a hacer, aprender a vivir juntos y aprender a ser. Es en el desarrollo de «aprender a hacer», exclusivamente, cuando el informe introduce el término "competencia» en el título del apartado De la cualificación a la competencia. En este apartado se explica que:

Aprender a conocer y aprender a hacer son, en gran medida, indisociables. Pero lo segundo está más estrechamente vinculado a la cuestión de la formación profesional: ¿cómo enseñar al alumno a poner en práctica sus conocimientos y, al mismo tiempo, cómo adaptar la enseñanza al futuro mercado de trabajo, cuya evolución no es totalmente previsible? (p. 99).

Apuntar, en primer lugar, que la «competencia» se vincula únicamente en el informe con el «saber hacer», y éste con la formación profesional. En segundo lugar, se trata de adaptar la enseñanza a un futuro mercado de trabajo imprevisible en el que: «la sustitución del trabajo humano por máquinas [...] acentúa el carácter cognitivo de las 
tareas, incluso en la industria, así como la importancia de los servicios en la actividad económica» (p. 99). Pero esto no será suficiente porque «el futuro de esas economías está supeditado a su capacidad de transformar el progreso de los conocimientos en innovaciones generadoras de nuevos empleos y empresas» (p. 99). Además indica que los empleadores, cada vez más, exigen no sólo una calificación o formación técnica y profesional, también competencias personales como «comportamiento social», aptitud de trabajo en equipo, iniciativa y capacidad de asumir riesgos, que constituyen lo que «los empresarios denominan a menudo "saber ser"» (p. IOo).

Más tarde, en el año 2000, el equipo encargado de diseñar el proyecto Tuning para los países de la Comunidad Europea tenía como cometido establecer unos «parámetros comunes», aunque «flexibles», para el diseño de las titulaciones a través de las competencias genéricas y las competencias específicas de área y sus correspondientes créditos. Sin embargo, en España muchos siguen preocupados por comprender el concepto de competencia y, sobre todo, por cómo evaluarla. González, Arquero y Hassall (20I4) identifican algunos factores que han obstaculizado el proceso de implantación, entre los que destacan la ambigüedad percibida por los profesores respecto de los conceptos y criterios de la formación por competencias, sobre todo en la etapa de codificación. En concreto, los profesores encuentran confusos los conceptos de competencia, capacidad y objetivos, así como la evaluación de las competencias.

\section{I. Algunos conceptos implicados en el diseño curricular por competencias}

Comenzamos por comparar la definición de los conceptos «competencia»y «resultados de aprendizaje» de la versión Tuning americana (20I2) y de la versión Tuning española (2006) a través de la Tabla I.

Tabla r: Comparación de la terminología entre el Tuning americano y el español

\begin{tabular}{|c|c|}
\hline Tuning EE. UU. & Tuning Europa versión española \\
\hline $\begin{array}{l}\text { Competency: puntos de referencia de do- } \\
\text { minio para las categorías de conocimiento y } \\
\text { aprendizaje dentro de una disciplina que se } \\
\text { incrementa de acuerdo al nivel de estudios. } \\
\text { Se extraen de la plantilla de los conceptos bá- } \\
\text { sicos, con competencia en cada nivel de gra- } \\
\text { do descrito en una breve declaración. Des- } \\
\text { criben los niveles de aprendizaje dentro de la } \\
\text { disciplina (p. 28). }\end{array}$ & $\begin{array}{l}\text { Competencia: se entienden como conocer } \\
\text { y comprender (conocimiento teórico de un } \\
\text { campo académico, la capacidad de conocer y } \\
\text { comprender), saber cómo actuar (la aplicación } \\
\text { práctica y operativa del conocimiento a cier- } \\
\text { tas situaciones), saber cómo ser (los valores } \\
\text { como parte integrante de la forma de perci- } \\
\text { bir a los otros y vivir en un contexto social) } \\
\text { (p. 28). [...] representan una combinación de } \\
\text { atributos (con respecto al conocimiento y sus } \\
\text { aplicaciones, aptitudes, destrezas y responsa- } \\
\text { bilidades) que describen el nivel o grado de } \\
\text { suficiencia con que una persona es capaz de } \\
\text { desempeñarlos. }\end{array}$ \\
\hline
\end{tabular}


Learning outcomes: declaraciones que describen la respuesta de los estudiantes al aprendizaje. [...] aíslan manifestaciones evaluables de aprendizaje que indican la habilidad con la que los estudiantes han dominado los conjuntos de conocimientos y procesos que componen una competencia determinada (p. 28).
Resultados de aprendizaje: son formulaciones de lo que el estudiante debe conocer, comprender o ser capaz de demostrar tras la finalización del proceso de aprendizaje. Pueden estar referidos a una sola unidad o módulo del curso o a un periodo de estudios [...]. Los resultados del aprendizaje especifican los requisitos mínimos para la concesión de un crédito (pp. 16-17).

Hay que señalar que, como reconoce Wagenaar (20I4), «Tuning fue el único y el primero en integrar los conceptos de competencias y resultados de aprendizaje» (p. 23), a lo que podemos añadir que fue también el primero en otorgar a las competencias un carácter formativo y evaluable en un ambiente distinto al que supuestamente van a ser utilizadas.

Para los americanos la competencia es aquello que el estudiante debe conocer y saber hacer (conocimientos y habilidades) en referencia a una disciplina, tomando en cuenta las posibles carreras profesionales de los egresados y según el nivel de estudios que esté cursando. En Europa, según Tuning (2006), se trata de «conocer, comprender, saber aplicar», "los valores que permiten "percibir" a los otros» y «vivir en un contexto social», etc., lo que deja amplio margen a la interpretación y complica la comprensión del concepto de competencia, que parece confundir con el de persona competente, aún más cuando el término «competencia» se trata como sinónimo de «resultado de aprendizaje»: «Cuando hablamos de los resultados de aprendizaje o de las competencias, nos referimos al conocimiento teórico, las habilidades analíticas, $[\ldots] »(\mathrm{p} .75)$.

Una diferencia de conceptos significativa reside en el concepto de «curso». En España asociamos este término al nivel (I. ${ }^{\circ}, 2 .^{\circ}, 3 .^{\circ}$ y $4 .^{\circ}$ curso). En EE. UU. «curso» se entiende como unidad independiente de aprendizaje con sus correspondientes créditos asociados. Los créditos, por tanto, se asocian a los cursos dirigidos a la adquisición de una o varias competencias, no a las asignaturas. Los niveles de los cursos (básico, avanzado, especializado, etc.) vienen determinados por la complejidad del contenido o habilidades a lograr o por los conocimientos y habilidades previos necesarios para seguir el curso. El resultado de la suma de los créditos de los diferentes cursos, algunos obligatorios y otros opcionales según la especialización elegida por el alumno, debe corresponderse con el mínimo exigido para la obtención del título.

\section{2. Los objetivos de aprendizaje y la evaluación: "Keep calm and use Bloom's taxonomy»}

Desde la perspectiva americana, las competencias no se evalúan directamente, puesto que eso es sólo posible en situaciones de la vida real. Lo que se evalúa son los resultados de aprendizaje que se estima que componen la competencia. Una buena declaración de un resultado de aprendizaje debe ser SMART ${ }^{3}$, es decir: I) centrada en el

Student-centered, Measurable, Action-oriented, Results-driven, Tailored to specfic degree levels. 
estudiante; 2) medible; 3 ) orientada a la acción; 4) orientada a los resultados; 5) ajustada al nivel de estudios: los objetivos deben escalarse según el nivel bachelor o máster.

En definitiva, una sentencia que describe un resultado de aprendizaje debe contener un verbo de acción, debe describir el resultado terminal del aprendizaje y debe ser medible. Conviene recordar en este punto la definición de Cagne (1965) de un objetivo de aprendizaje: «[...] una declaración verbal que se comunica con fiabilidad a cualquier persona (que conoce las palabras de la declaración como conceptos) [...] el conjunto de circunstancias que identifica una clase de actuaciones humanas» (p. 243). Esta «declaración verbal» debe, según el mismo autor, contener al menos estos componentes:

I) Un verbo que indique la acción observable: por ejemplo, dibujar, identificar, reconocer, calcular; no sirven: ver, conocer, comprender, etc.

2) Una descripción del tipo de estímulo que será respondido, por ej., «dada la siguiente ecuación $a b+a c=a(a+c) »$.

3) Una palabra o frase indicando el objeto usado para la acción por el individuo, a menos que esté implícito en el verbo (por ej. si el verbo es «dibujar» esta frase podría ser «con un tiralíneas»; si la palabra es «indicar» la palabra podría ser «oralmente»).

4) Una descripción de la clase de respuestas correctas (por ej., «un ángulo recto» o «la suma» o «el nombre de la regla»).

El informe Tuning American High Education (2012) recomienda, además, la taxonomía de Bloom que fue presentada en su obra Taxonomy of educational objectives: The classification of educational goals (1956) ${ }^{4}$, cuyas categorías eran: Conocimiento, Comprensión, Aplicación, Analisis, Síntesis y Evaluación, descompuestas en subcategorías (excepto Aplicación) y ordenadas de la más simple a la más compleja y de lo más concreto a lo más abstracto. El dominio de la categoría más simple (conocimiento) era necesario para el dominio de la siguiente, por lo que se asumía una jerarquía acumulativa de las categorías (Krathwohl, 2002). Según el autor, cuando se extendió el uso entre los profesores de la taxonomía original, se evidenció que la mayoría de las cuestiones de las pruebas de evaluación se situaban en la categoría «recordar», a lo sumo «comprender». Krathwohl (2002) revisa la taxonomía original de Bloom intentando subsanar sus debilidades de manera que el objetivo de aprendizaje recoja tanto el contenido a aprender como el proceso cognitivo implicado, o subcategorías, dando mayor énfasis a estas últimas. En esta nueva taxonomía, Krathwohl (2002) sitúa la categoría crear, que no existía en la original, en la cúspide de la pirámide cognitiva y a las demás por debajo en el orden siguiente: evaluar, analizar, aplicar, comprender y recordar (ver Figura 2).

Esta nueva clasificación es la que recomienda el informe Tuning americano (sin hacer referencia a Krathwohl sino a Bloom), aclarando que las categorías no se corresponden con los niveles de los grados, sino que los estudiantes se mueven a través de todas ellas cada año.

4 La taxonomía original fue desarrollada posteriormente en los dominios psicomotor: Simpson, E. (1972) The Classification of Educational Objectives in the Psychomotor Domain: The Psychomotor Domain Vol. 3. Washington dc: Gryphon House y afectivo: Krathwohl, D. R.; Bloom, B. S. y Masia, B. B. (I964) Taxonomy of educational objectives: Handbook II: Affective domain. New York: David McKay Co. 
DOS RECORRIDOS DIFERENTES PARA LA IMPLANTACIÓN DE LAS COMPETENCIAS

EN LA UNIVERSIDAD: ESPAÑA Y EE. UU.

ILUMINADA SÁNCHEZ DOMENECH Y MARIANO RUBIA AVI

Figura 2: Estructura de la dimensión del proceso cognitivo en la Taxonomía Revisada

1. Recordar: recuperar el conocimiento relevante desde la memoria a largo plazo

1.1. Reconocer

1.2. Recordar

2. Comprender: determinar el significado de los mensajes instruccionales, incluyendo la comunicación escrita, oral y gráfica.

2.1. Interpretar

2.2. Ejemplificar

2.3. Clasificar

2.4. Resumir

2.5. Inferir

2.6. Comparar

2.7. Explicar

3. Aplicar: llevar a cabo o utilizar un procedimiento en una situación dada

3.1. Ejecutar

3.2. Implementar

4. Analizar: descomponer el material en sus partes constituyentes y detectar cómo las partes se relacionan unas con otras para formar una estructura global o propósito

4.1. Diferenciar

4.2. Organizar

4.3. Atribuir

5. Evaluar: hacer juicios basados en criterios y normas

5.1. Comprobar

5.2. Criticar

6. Crear: colocar elementos juntos para formar un todo nuevo coherente o hacer un producto original

Fuente: Adaptado de Krathwohl (2002).

El autor, además, propone una «sencilla tabla» para facilitar la selección de objetivos de un curso o competencia, que contiene las categorías principales y las subcategorías. La siguiente tabla (Tabla 2) es un ejemplo para la categoría conocimiento en la que hemos incluido algunos verbos descriptores de la acción bajo la categoría cognitiva correspondiente.

TABLA 2: Tabla ejemplo para la determinación de objetivos de conocimiento

\begin{tabular}{|l|l|l|l|l|l|l|}
\hline & $\begin{array}{l}\text { Recordar } \\
\text { Define, } \\
\text { describe, } \\
\text { Dimensión } \\
\text { Conocimiento } \\
\text { completa, } \\
\text { selecciona, } \\
\text { enumera, } \\
\text { marca... }\end{array}$ & $\begin{array}{l}\text { Comprender } \\
\text { Compara, } \\
\text { concluye, } \\
\text { contrasta, } \\
\text { explica, } \\
\text { resume, } \\
\text { relaciona... }\end{array}$ & $\begin{array}{l}\text { Aplicar } \\
\text { Interpreta, } \\
\text { resuelve, } \\
\text { demuestra, } \\
\text { calcula, } \\
\text { diseña, } \\
\text { elabora... }\end{array}$ & $\begin{array}{l}\text { Analizar } \\
\text { Diferencia, } \\
\text { categoriza, } \\
\text { clasifica, } \\
\text { relaciona... }\end{array}$ & $\begin{array}{l}\text { Evaluar } \\
\text { Compara, } \\
\text { jerarquiza, } \\
\text { valora, } \\
\text { juzga, } \\
\text { critica... }\end{array}$ & $\begin{array}{l}\text { Crear } \\
\text { Diseña, } \\
\text { construye, } \\
\text { inventa, } \\
\text { elabora, } \\
\text { reelabora... }\end{array}$ \\
\hline Factual & Objetivo I & & & & & \\
\hline Conceptual & & Objetivo 2 & & & & \\
\hline Procedimental & & & Objetivo 3 & & Objetivo 4 & \\
\hline Metacognitivo & & & & & & Objetivo 5 \\
\hline
\end{tabular}

Fuente: Adaptada de Krathwohl (2002).

En el Tuning europeo (2006) la relación entre competencia y objetivos de aprendizaje no es tan explícita. El término utilizado es «resultado del aprendizaje» que puede corresponderse con: «conocer, comprender o ser capaz de demostrar» (p. 8) según la definición. Lo que dejaría fuera otros procesos cognitivos superiores como analizar, evaluar y crear. Para los creadores del informe europeo, Tuning se basa en una serie de 
características entre las que se encuentran: «los resultados de aprendizaje correspondientes formulados en términos de competencia» (p. I3I), lo que viene a suponer partir de los resultados de aprendizaje a lograr para, después, reformularlos en términos de competencias, de manera que se haga visible lo que el alumno sabrá hacer (al menos en potencia) una vez superados los objetivos de aprendizaje.

Más adelante para definir las competencias específicas, el informe Tuning europeo utiliza términos similares a los tradicionalmente ligados al concepto de objetivo de aprendizaje (conocimientos, habilidades, actitudes y valores): «Todos los programas de titulación desarrollan competencias específicas del área de conocimiento, esto es, conocimientos, capacidades, habilidades y valores...» (p. I43), lo que contribuye a la confusión, al establecer una equivalencia implícita entre las competencias específicas y los objetivos de aprendizaje.

Pero tampoco contribuyó demasiado a esclarecer la diferencia o relación entre competencias y objetivos de aprendizaje la Agencia Nacional de Evaluación de la Calidad y Acreditación (ANECA), que en su Guía de Apoyo para la elaboración de la memoria de títulos oficiales universitarios (grado y máster) en su ANEXo v. Orientaciones para la definición de competencias, dice: «Una competencia ha de recoger los siguientes dos elementos» (p. 84) que, como se puede comprobar, coinciden con la definición de objetivo de aprendizaje de Cagne (1965) que vimos anteriormente:

- «Un verbo activo»: por ejemplo: definir, describir, identificar, interpretar, relacionar, discutir, aplicar, demostrar, solucionar, etc., que servirá para estimar o jerarquizar el grado de complejidad de cada una de las competencias.

- «La descripción del objeto o de la acción y el contexto en el que se aplica»: se refiere al campo disciplinario en el que se fundamenta, por ejemplo, «traducir e interpretar textos latinos».

Además, ANECA incluye «un listado de los verbos activos (de menor a mayor requerimento intelectual en función de sus objetivos)»(p. 84). Ese listado coincide con la primera taxonomía de Bloom (1956): conocer, comprender, aplicar, analizar, sintetizar y evaluar, aunque no hace referencia al autor. Recientemente (2014/2015) ANECA ha publicado en su Web la Guía de apoyo para la redacción, puesta en práctica y evaluación de los RESULTADOS DEL APRENDIZAJE. En su punto 3.I.2. titulado «Una herramienta útil para redactar resultados del aprendizaje», se basa en la taxonomía de Bloom y Krathwohl. Las competencias quedan fuera del diseño curricular que se estructura en torno a tres elementos: objetivos de aprendizaje, actividades formativas y métodos de evaluación.

\subsection{El proceso de concreción curricular}

En las universidades estadounidenses la formulación en términos de competencias se hace a partir de una relación de tareas imprescindibles o esenciales, consensuadas entre los miembros de la facultad, teniendo en cuenta los posibles itinerarios profesionales, que deben estar concretadas en resultados de aprendizaje medibles. La independencia de los cursos, con respecto a las asignaturas y a los cursos (como aquí los entendemos), permite organizar los grados en torno a las competencias. Un curso, por tanto, puede estar dirigido a la adquisición de una o varias competencias por parte 
del estudiante y en diferentes niveles de complejidad y especialización a través de la titulación y en él pueden intervenir profesores de distintas especializaciones.

Tuning en Europa (2006) recomienda igualmente comenzar el diseño del programa a partir de los objetivos/resultados de aprendizaje. Estos son los pasos que propone:

3. Descripción de los objetivos del programa y de los resultados de aprendizaje (en términos de conocimientos, comprensión, habilidades y capacidades) que deberán cumplirse.

4. Identificación de las competencias genéricas y específicas de cada área que deberían alcanzarse en el programa.

5. Traducción al plan de estudios: contenido (temas que deberán cubrirse) y estructura (módulos y créditos).

6. Traducción a unidades y actividades educativas para alcanzar los resultados de aprendizaje definidos (p. I4).

No se especifica en este proceso qué elementos, si competencias u objetivos, se «traducen» a unidades y actividades educativas. Parece que en España se ha tendido a partir de la formulación de las competencias en el proceso contrarreloj de homologar los nuevos grados. Veamos gráficamente cómo ha quedado el proceso de diseño curricular en estas universidades españolas (Figura 3) y abajo cómo se lleva a cabo en EE. UU. (figura 4).

FiguRa 3: Proceso de diseño curricular por competencias en España

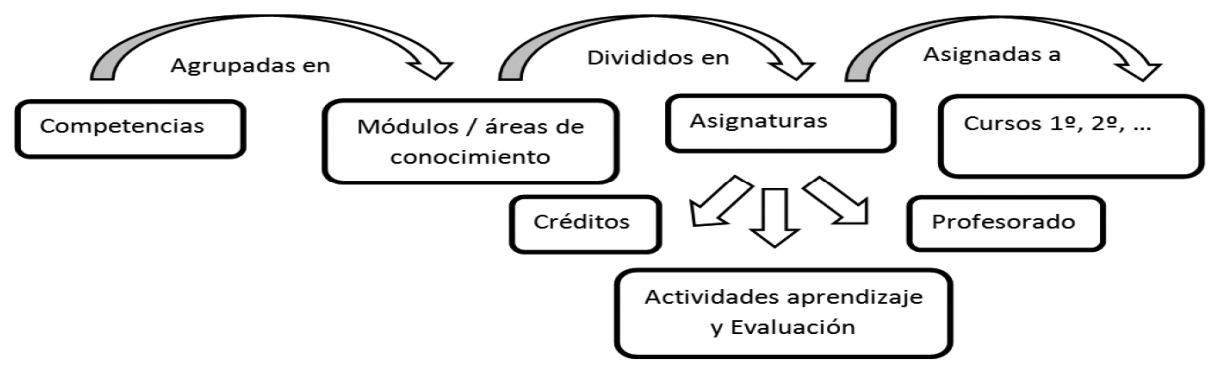

Figura 4: Proceso de diseño curricular por competencias en EE. UU.

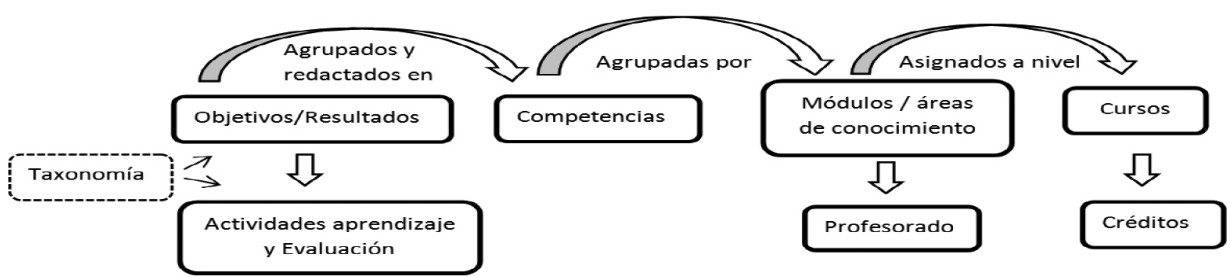

Por tanto, podríamos decir que en España también nos encontramos en un «modelo híbrido», pero en este caso entre la estructura curricular en torno a competencias y la estructura curricular en torno a asignaturas. 


\subsection{La metodología activa a través de Dewey y Bruner}

La versión americana de Tuning no insiste demasiado en las metodologías que fomentan el método de investigación y el aprendizaje autónomo y que se centran en la actividad del estudiante, puesto que son parte de la identidad de Universidad americana (ver punto del informe americano Tuning, p. 6 de este documento). La realidad de las aulas españolas era bastante diferente, y de ahí, suponemos, la euforia innovadora que suscita el modelo:

Así pues, fruto de la implantación de las competencias, las clases deberían dejar de ser magistrales, como mecanismo de transmisión de conocimientos del profesor al estudiante, para aplicar la combinación sucesiva de diferentes metodologías docentes y actividades que permitan ejercitar las diferentes competencias: tutorías, seminarios, trabajos de investigación... (Delgado, Borge, García Oliver y Salomón, 2005: 35).

Fue Dewey (1859-1952) quien cuestionó la dicotomía entre el aprendizaje puramente memorístico y académico frente al práctico y profesional. En la práctica esta postura conciliadora de Dewey se resuelve a través del diseño de actividades problemáticas que involucren tanto conocimientos teóricos como prácticos, y tanto procedentes de la esfera científica, histórica o artística, como de la social y la industrial, con finalidad democráticamente transformadora del orden existente. Para Dewey (1938) los problemas son el estímulo para pensar; además, para que el crecimiento se produzca debe existir una dificultad en la experiencia presente que debe ser superada a través del ejercicio de la inteligencia. La escuela ha de ser el embrión de la sociedad democrática (Dewey, I897), «tiene el poder de modificar el orden social» (Dewey, 1909: 3). En esta medida, la educación es esencial para la democracia y su supervivencia depende de un ciudadano educado en los derechos y responsabilidades de una democracia. La formación moral de las escuelas es otra de las preocupaciones de Dewey, si no la principal. Y es que para Dewey la vida social democrática se sustenta en los valores morales de los individuos.

Los conceptos entendidos hoy como metodologías activas de aprendizaje y que incluyen: aprendizaje por descubrimiento (discovery method), el método de investigación (inquiry method) o el aprendizaje basado en problemas (problem-solving learning) provienen de la corriente cognitiva de los años 6o. Uno de los mayores exponentes de estas metodologías es Jerome Bruner. A través de este autor podemos situarnos en el contexto del surgimiento del constructivismo. En los 6o, según Bruner (1960), la preocupación de las instancias educativas y científicas era cómo conseguir hacer llegar a los niños y jóvenes la ciencia, no sólo los contenidos de la ciencia, es decir, aprender sobre ciencia, sino el método científico, se trataba de: «mejorar la calidad y los objetivos intelectuales de la educación pero sin abandonar el ideal de que la educación debe servir como un medio para formar ciudadanos equilibrados para una democracia» (p. 2).

Bruner (1966) entiende por «descubrimiento» «todas las formas de obtener conocimiento por uno mismo a través del uso de nuestra propia mente» (p. I). No es un simple encuentro con verdades aisladas, sino que implica reorganizar o transformar las evidencias, implica ser capaz de ir más allá de la evidencia «reensamblando» o reorganizando las nuevas ideas adicionales o, simplemente, las evidencias que ya existen. Bruner (196I) distingue entre el modo expositivo y la enseñanza en modo hipotético: 
En el primero, las decisiones concernientes a la forma, ritmo y estilo de exposición están principalmente determinadas por el profesor como expositor; el estudiante es el oyente [...]. En el modo hipotético, el profesor y el estudiante se encuentran en una posición más cooperativa [...]. El estudiante no es un oyente atado a la silla, sino que toma parte en la formulación y a veces debe jugar el papel principal en él (Bruner, I96r: 2).

El modo hipotético (investigación, resolución de problemas, descubrimiento) lleva a los estudiantes a implicarse en «actos de descubrimiento», un proceso al que Bruner (196r: 2) atribuye cuatro beneficios: I) incremento de las capacidades intelectuales, 2) desplazamiento desde las recompensas extrínsecas hacia las intrínsecas, 3) aprender las heurísticas del descubrimiento y 4) hacer el material más fácilmente accesible en la memoria. ¿Pero cómo se aprende a investigar, a descubrir por uno mismo, a solucionar problemas? Para Bruner sólo hay una respuesta: investigando, descubriendo y solucionando problemas, es decir, practicando. Practicar el descubrimiento por uno mismo no sólo enseña a encontrar relaciones y regularidades, sino que debe enseñar también a utilizar la información relevante de forma que sirva para resolver problemas (Bruner, 196I) y a desechar la que no sirve.

Bruner (1966) defendía el uso de la competencia. Sin embargo, lo que distingue el concepto de competencia en su concepción actual, como objetivos educativos prefijados (blindados al profesor) para la formación profesional, del uso que los teóricos cognitivos le daban al concepto es el de la motivación intrínseca para aprender. La competencia es entendida como una interacción efectiva con el ambiente. Bruner afirma que hay ciertas formas de actividad como la autonomía, la curiosidad y el juego, que sirven para activar y desarrollar la motivación de competencia, que funcionan como una fuerza interna impulsora del comportamiento. Knowles (1980), educador de adultos y considerado el padre de la andragogía en EE. UU., adopta el concepto de competencia como esa fuerza motivadora que ha comprobado que da resultado con sus estudiantes, al sustituir los objetivos del curso y sus horas correspondientes de permanencia en el aula por la descripción de capacidades a lograr, lo más autónomamente posible y a través de la experimentación.

\section{Conclusiones}

El constructivismo data de principios del siglo xx, cuyo más insigne precursor fue Dewey. Las «nuevas metodologías activas de aprendizaje» proceden de la corriente cognitiva de los años 60 y los objetivos conductuales de los 50 . Las competencias profesionales proceden de la psicología laboral u organizacional americana que comienza en los so. Nada nuevo bajo el sol, excepto que todo eso se pone ahora al servicio de la formación para el empleo globalizado. Un nuevo componente lo constituyen las nuevas tecnologías de la información (TIC). Aunque Area (2006) advierte que las propuestas novedosas que se basan en el uso de las TiC y la Red «no son más que una relectura y aplicación de esos veteranos principios pedagógicos y de sus propuestas metodológicas [de la Escuela Nueva] a los nuevos tiempos digitales» (p. 4).

Si algo dejan claro Tuning y ANECA con respecto al diseño curricular por competencias es que, en primer lugar, éstas deben ser evaluables y, en segundo lugar, para que sean evaluables deben estar definidas en términos de resultados de aprendizaje, que en la práctica vienen siendo los objetivos conductuales de toda la vida. 
En el modelo de competencias trasladado a la educación, se consideran ambos enfoques, el conductista y el constructivista, complementarios. Ambos deben estar presentes en el diseño curricular: (I) la definición de los objetivos de aprendizaje son conductistas con el fin de ser evaluables y medibles; (2) se recomienda que los procesos de enseñanza-aprendizaje sean constructivistas si se pretende que el futuro egresado sea capaz de seguir aprendiendo de forma autónoma a lo largo de la vida, de generar nuevo conocimiento y de emprender y crear nuevas empresas y empleos en «un mercado laboral global saturado de trabajadores bien educados de bajo coste» (Brown, Lauder y Ashton, 2oII: I2).

A nuestro parecer, una de las causas de la dificultad de la implantación del modelo de competencias en España es que se asume que los objetivos de aprendizaje -que se clasificaban en conocimientos, habilidades, intereses, actitudes y valores- deben ser totalmente sustituidos por las competencias cuando, en realidad, son necesariamente complementarios. Podemos decir que las competencias no «informan», utilizando el término de Bruner (1960: I5) suficientemente ni a profesores ni alumnos. Para que eso suceda, sería necesario partir de unos objetivos comportamentales, es decir, observables y medibles, que describen la competencia y que sirven como criterio para la selección de las actividades de aprendizaje y las técnicas e instrumentos de evaluación de forma coherente. Pero entonces cabe preguntarse, ¿qué propósito tiene reformular los objetivos en competencias? Redactar los objetivos o los resultados del aprendizaje, reagruparlos por dominios y formularlos en términos de competencias permite:

- Facilitar a los empleadores la correspondencia entre el título y el puesto de trabajo, es decir, la señalización de los egresados.

- Identificar competencias transferibles de una titulación a otra y entre países.

- Rendición de cuentas.

- Facilitar la movilidad laboral para un mercado de trabajo global.

- Promover la reflexión de los responsables académicos sobre la aplicación práctica de los contenidos a las potenciales salidas profesionales de la titulación, es decir, su transferencia al mercado laboral.

Las taxonomías de objetivos de aprendizaje son necesarias herramientas, como indica ANECA, en el diseño curricular orientado a competencias impuesto, por razones prácticas:

- Facilitan la selección y clasificación de los objetivos descriptores de los aprendizajes deseados en los distintos dominios.

- Permiten la congruencia entre objetivos de aprendizaje, actividades de enseñanza-aprendizaje y evaluación.

- Facilitan la selección del tipo de metodología de evaluación, sus criterios y, en las pruebas escritas, el tipo y la redacción de las cuestiones.

- Informan a los alumnos sobre el tipo de aprendizaje que se espera de ellos.

La estructura curricular de cursos de las universidades americanas facilita la transdisciplinareidad en torno a situaciones problemáticas (López, 20II) que en España se reclama para nuestras universidades, sin embargo, ha sido necesaria siempre. En España la falta de flexibilidad de la estructura por asignaturas debe ser compensada con el trabajo colaborativo de profesores para el diseño de actividades problemáticas de 
aprendizaje holístico e integrador en la que deban ponerse en juego conocimientos y habilidades adscritas en el plan de estudios a las distintas asignaturas. Los aprendizajes transversales (lo que aquí se entiende como «saber ser» y «saber convivir con otros») como la democracia, la participación activa en el entorno, el pensamiento crítico, la autonomía, la creatividad, la tolerancia, el trabajo en equipo no pueden ser reducidos a competencias comportamentales dependientes de una asignatura o de varias, ni siquiera de la titulación, sino que deben formar parte de la cultura institucional. Este podría ser un antídoto posible contra la instrumentalización de las virtudes humanas -convertidas en «habilidades interpersonales laborales» por el lenguaje del modelo competencial-, como arma para la eficacia profesional.

Las competencias son sólo la parte visible, el escaparate, de las titulaciones, una referencia que tiene como fin servir de guía para la selección de aquellos objetivos/ resultados de aprendizaje que posean utilidad en el mercado laboral global, sin que esto deba suponer excluir los que se dirijen a educar «ciudadanos equilibrados para una democracia» (Bruner, 1960: 2). No está de más recordar la siguiente recomendación del informe Delors:

[...] ninguno de los elementos fundamentales del conocimiento -aprender a conocer, aprender a hacer, aprender a ser, aprender a vivir juntos- debe descuidarse en provecho de los demás (p. I8I).

En definitiva, en caso de comulgar con el modelo, cambios de mayor calado deben acometer las universidades españolas, comenzando por el diseño curricular. En caso contrario, «Hay mucho y valioso en las tradiciones pedagógicas europeas del aprendizaje liberal/humanista y del crítico. Sería una locura criminal pura permitir que estas sean destruidas por la adopción acrítica de un modelo conductista fallido de competencia» (Hyland, 2006: 7).

\section{Bibliografía}

Aneca (s. f.) Guía de Apoyo para la elaboración de la memoria de titulos oficiales universitarios (grado y máster). Recuperado el is de abril de 20I5, de http://www.aneca.es/Programas/verifica/Verificacion-de-Grado-y-Master/Protocolos-de-evaluacion-y-documentos-de-ayuda.

ANECA (20I4/20I5) Guía de apoyo para la redacción, puesta en práctica y evaluación de los RESULTADOS DEL APRENDIZAJE. Recuperado el 20 de abril de 20I5, de http://www.aneca.es/ Documentos-y-publicaciones/Otros-documentos-de-interes/Otras-guias-y-documentos-de-evaluacion.

Area, M. (2006) Hablemos más de métodos de enseñanza y menos de máquinas digitales: los proyectos de trabajo a través de la www. Cooperació Educativa (MCEP), 79, Monográfico «La investigación escolar salta a la Red», 26-32.

Association of American Colleges \& Universities (Aac\&U, 20i6) Recuperado el 4 de abril de 2016, de http://www.aacu.org/leap/what-is-a-liberal-education.

Bloom, B. S. (ed.); Engelhart, M. D.; Furst, E. J.; Hill, W. H. y Krathwohl, D. R. (1956) Taxonomy of educational objectives: The classification of educational goals. Handbook I: Cognitive domain. New York: David McKay.

Boyatzis, R. E. (1982) The competent manager: A model for effective performance. Nueva York: John Wiley \& Sons. 
Boyatzis, R. E. (2008) Competencies in the 2ist century. Journal of Management Development, 27 (I), 5-I2 Doi: 10.1108/02621710810840730.

Boyatzis, R. E.; Goleman, D. y Rhee, K. (2000) Clustering competence in emotional intelligence: Insights from the emotional competence inventory (ECI). En R. BAR-ON y J. D. A. PARKer (eds.), Handbook of Emotional Intelligence (pp. 343-362). San Francisco: Jossey-Bass.

Brown, P.; Lauder, H. y Ashton, D. (20II) The Global Auction: The Broken Promises of Education, Jobs and Income. Oxford: Oxford University Press.

Bruner, J. S. (1960) The Process of Education. Cambridge, Mass: Harvard University Press.

Bruner, J. S. (196I) The act of discovery. Harvard Education Review, 3I, 2I-32.

Bruner, J. S. (1966) Toward a Theory of Instruction (pp. I25-I27). Cambridge, Ma: Harvard University Press.

CAel (20I2) Competency-Based Degree Programs in the U.S. Postsecondary Credentials for Measurable Student Learning and Performance. Recuperado el is de abril de 20I5, de http:// www.cael.org/pdfs/20I2_competencybasedprograms.

Cagne, R. M. (1965) The conditions of learning. New York: Holt, Rinehart and Winston.

Delgado, A. M.; Borge, R.; García, J.; Oliver, R. y Salomón, L. (2005) Competencias y diseño de la evaluación continua y final en el Espacio Europeo de Educación Superior. Programa de Estudios y Análisis n. ${ }^{\circ}$ Ref.: EA2005-0054. Dirección General de Universidades. Ministerio de Educación y Ciencia (España).

Dewey, J. (I897) My Pedagogic Creed. The School Journal, LIV (3), 77-80.

Dewey, J. (1909) Moral principles in Education. Houghton Mifflin Company. The Riverside Press Cambridge.

Dewey, J. (1938) Experience and Education. New York: Macmillan.

Díaz Barriga, A. (2009) Diseño curricular por competencias. Apertura de temas que significan un regreso a los viejos problemas de la educación. Ponencia presentada en el $X$ Congreso Nacional de Investigación Educativa Veracruz. Veracruz, 2I al 25 de septiembre de 2009.

Flanagan, J. C. (1954) The critical incident technique. Psychological bulletin, 5I (4), 327-358.

Goleman, D. (1998) La práctica de la inteligencia emocional. Barcelona: Kairós.

González, J. M.; Arquero, J. L. y Hassall, T. (20I4) Consolidacion de la formación por competencias en la universidad espanola: Estudio de un caso. Educacion XXI, I7 (2), I45-I68. Doi: I0.5944/educxxi.17.2.11483.

Hager, P. (2006) Some conceptual questions about the tuning project. Prospero I2, (I), 3I-38.

Hodge, S. (2007) The origins of competency-based training. Australian Journal of Adult Learning, 47 (2), 179-209.

Hyland, T. (2006) Swimming against the tide: reductionist behaviourism in the harmonisation of European higher education systems. Education: Journal Articles. Paper 9.

IEBC (2012) Tuning American Higher Education: The Process. Recuperado el 25 de abril de 20I4, de www.iebcnow.org.

Knowles, M. S. (1980) The modern practice of adult education: From pedagogy to andragogy. Englewoods ClifF, NJ: Cambridge Adult Education.

KrATHWOHL, D. R. (2002) A Revision of Bloom's Taxonomy: An Overview. Theory into practice, 4I (4), 2I2-2I8.

Le, C.; Wolfe, R. y Steinberg, A. (2014) The Past and the Promise: Today's Competency Education Movement. Students at the Center: Competency Education Research Series. Boston, MA: Jobs for the Future.

López, J. I. (2OII) Un giro copernicano en la enseñanza universitaria: formación por competencias. Revista de Educación, 356, septiembre-diciembre 20II, 279-301. Doi: 10-4438/1988-592XRE-20IO-356-040.

McClelland, D. C. (1973) Testing for competence rather than intelligence. American Psychologist, 28 (I), I-I4. 
Morrison, P. (1979) Guidelines for the organization and administration of competency-based vocational business education programs for adults. Department of Education Commonwealth of Pennsylvania. ERIC: I773IO.

Nussbaum, M. (2010) Sin Fines de Lucro. Por qué la democracia necesita de las humanidades. Madrid: Katz.

SolÀ Gussiner, P. (2009) ¿Cómo debiera ser la educación superior de adultos? [Recetas para conjurar el virus reduccionista/utilitario que nos llega en forma de teoría de las competencias profesionales]. En P. L. Moreno Martínez y C. Navarro García (coords.) Perspectivas históricas de la educación de personas adultas. Efora, vol. 3, n. ${ }^{\circ}$ I. Universidad de Salamanca. Fecha de consulta: 26/04/2015.

Tuning Educational Structures in Europe (2006) La contribución de las universidades al proceso de Bolonia. Una introducción. Recuperado el is de abril de 20I5, de http://www. deusto-publicaciones.es/deusto/pdfs/tuning/tuningi2.pdf.

unesco (1996) La educación encierra un tesoro. Informe a la Unesco de la Comisión Internacional sobre la educación para el siglo xxi. Delors, J. (pres.); Al Mufti, I.; Amagi, I.; R. Fay Chung; Geremek, B.; Gorham, W.; Kornhauser, A.; Manley, M.; Padrón, M.; Savané, M. A.; Singh, K.; Stavenhagen, R.; Won, M. y Nanzhao, Z. Madrid: Santillana.

WagenaAR, R. (20I4) Competences and learning outcomes: a panacea for understanding the (new) role of Higher Education? Competence-based learning: a global perspective. Volume I, Issue No. 2 May. Tuning Journal for Higher Education. 